\title{
Zum Verständnis von Converging Technologies
}

\section{Zugänge zu einem Zauberwort}

\author{
Aktuelle Diskussionen in Wissenschaft und \\ Technik kreisen immer wieder um die so ge- \\ nannten konvergierenden Technologien. Auf- \\ grund der vielfältigen thematischen Bereiche \\ und den Bezügen zu Wissenschaft wie auch \\ Technik ist eine Definition schwierig. Wie kann \\ dieser Begriff gefasst werden? \\ Von Torsten Fleischer und Michael Rader
}

D er Begriff Converging Technologies tauchte zuerst im Titel einer von der US-amerikanischen National Science Foundation und dem Department of Commerce gesponserten Workshop-Publikation auf (Roco/Bainbridge 2002). In dieser wird der Verschmelzung der Disziplinen Nanotechnologie sowie Bio- und Informationstechniken mit den Erkenntnissen der Kognitionswissenschaften eine zentrale Bedeutung beigemessen.

Dadurch, so ihre Protagonisten, erschlössen sich technische Ansätze, bisher biologisch begrenzte Fähigkeiten des Menschen erweitern und verbessern zu können. Die Publikation formuliert schon in ihrem Titel eine explizite Zielfunktion: „Converging Technologies for Improving Human Performance“ (Roco/Bainbridge 2002).

\section{Verbesserung der menschlichen Leistung}

Die Nanotechnologie wird im US-amerikanischen Kontext und in der Folge als die Schlüsseltechnologie betrachtet, durch die viele Entwicklungen im Rahmen von Converging Technologies überhaupt erst möglich werden. Auch die Bedeutung der Neurowissenschaften wird betont, ist dies doch einerseits die Disziplin, die den anderen drei Technologien eine Zielrichtung für deren Forschung vorgibt, und macht sie andererseits durch eigene Forschungsergebnisse bestimmte visionäre Anwendungen im Bereich Converging Technologies überhaupt erst vorstell- und umsetzbar. Bis heute ist allerdings eine Übertragung der zum Teil weitreichenden Visionen in konkrete Forschungsprojekte nur ansatzweise sichtbar.

Sicher wird in einer ganzen Reihe von Ländern an der Entwicklung von Techniken gearbeitet, die dem Oberbegriff Converging Technologies, kurz CT, zuzuordnen wären. Selten geschieht dies aber als Konsequenz von übergreifenden Forschungsstrategien zu diesem Bereich. Verbunden mit dem forschungspolitischen Konzept Converging Technologies taucht in einer Reihe von Dokumenten zudem die Vision einer neuen Renaissance der Wissenschaft auf, die vom Verständnis der Struktur und des Verhaltens der Materie auf der Nanoebene bis zum menschlichen Gehirn reicht.

\section{Amerikanische und europäische Sichtweisen}

Forschungspolitische Akteure der Europäischen Union reagierten zeitnah auf die US-amerikanischen Aktivitäten. Beginnend im Jahr 2003 wurde eine Reihe von Expertengruppen einberufen. Diese legten in der Folge Berichte vor, die sich in Abgrenzung zum US-amerikanischen Konzept Converging Technologies nicht nur auf die vier Schlüsseltechnologien beschränkten, sondern auf die Bedeutung weiterer Disziplinen aus dem Bereich der Sozial- und Geisteswissenschaften verwiesen und hinsichtlich gesellschaftlicher Anwendungsfelder den offenen Charakter der CT betonten. Dabei unterstreicht und problematisiert insbesondere der so genannte Nordmann-Bericht das Thema der Interdisziplinarität, das als Schlüssel zur Realisierung von Konvergenzvisionen betrachtet wird (HLEG 2004). In den Berichten der Expertengruppen wird der Förderung von Converging Technologies eine hohe strategische Priorität auf forschungspolitischer Ebene eingeräumt, da diese vor allem im Wettbewerb mit den USA als entscheidend für die zukünftige Stellung der Europäischen Union (EU) in der Welt angesehen werden. Es ist allerdings festzustellen, dass diese Bekenntnisse bis zum jetzigen Zeitpunkt nur selten Niederschlag in konkreten europäischen Forschungsprogrammen fanden. Immerhin ist vor einigen Jahren eine Abteilung der EU-Forschungsdirektion in „Nano- and converging sciences and technologies" umbenannt worden.

Neben Aktivitäten auf supranationaler europäischer Ebene entwickeln die einzelnen Mitgliedsstaaten der EU nationale Ansätze, die sich zum Teil sehr voneinander unterscheiden. Ohne dies hier im Einzelnen länderspezifisch darstellen zu können, bleibt festzuhalten, dass es in einer Reihe von Mitgliedstaaten nationale Forschungsprojekte gibt, die sich mit Entwicklungen im Bereich Converging Technologies beschäftigen, ohne dass diese einer Gesamtstrategie oder einer Vision zu diesem Forschungsbereich zugeordnet werden können (Quendt et al. 2007).

Aktuell scheint es zudem so zu sein, dass nach einigen Jahren der rhetorischen Euphorie vor allem in der US-amerikanischen Forschungspolitik ein Prozess der Normalisierung einsetzte. Einige amerikanische Kollegen vertraten hierzu sogar die Auffassung, dass die Rezeption des Konzepts der CT und $\rightarrow$ 


\section{„Das Verständnis von Converging Technologies reicht von einer engen ingenieurwissenschaftlichen Rahmung bis zu einem Avantgarde-Label“}

Schwerpunkt befasst sich vor allem mit ethischen und sozialen Fragen zur Verbesserung menschlicher Leistungsfähigkeit. Dabei reicht das Spektrum von Themen der praktischen Bioethik neuronaler Implantate oder pharmakologischen Enhancements bis hin zur Rekonstruktion des Menschen oder seiner posthumanen Zukunft. Ergänzt werden diese Arbeiten durch Untersuchungen zu den kulturellen, historischen und politischen Kontexten solcher Visionen und den damit verbundenen Diskursen (z. B. Grunwald 2007). Ein dritter Strang befasst sich vornehmlich mit Fragen neuer Modi der Inter- und Transdisziplinarität in den Wissenschaften, der gesellschaftlichen Einbettung von Wissenschaft und Technik sowie empirischen Aspekten von Prozessen der technowissenschaftlichen Konvergenz (z. B. Schmidt 2007).

seine Auseinandersetzung damit in Europa sehr viel intensiver gewesen seien als in den USA selbst. Dies sei durch Missverständnisse bei der Interpretation seiner forschungspolitischen Bedeutung sowohl für die USA als auch für Europa noch gefördert worden.

\section{Debatte über Anwendungen}

Dessen ungeachtet hat sich vor allem im akademischen Bereich eine Debatte über Converging Technologies und insbesondere ihre Anwendung mit dem Ziel des menschlichen Enhancement entwickelt. Eine Frage, die diese Diskussionen seit geraumer Zeit begleitet, ist die nach dem Gegenstandsbereich. Sie kann durch folgende Fragen eingegrenzt wird:

- Handelt es sich bei CT primär um neue technische Entwicklungen zur Wiederherstellung und Verbesserung von motorischen, sensorischen oder kognitiven Fähigkeiten von Individuen?

- Steht CT nur als neutral anmutendes Synonym für „Technologies for Human Enhancement"?

- Was beschreibt CT im Kern? Ein weiteres forschungspolitisches Programm, das im Wettbewerb um Aufmerksamkeit der Forschungsförderer mit teilweise überzogenen visionären Vorstellungen und Versprechungen hinsichtlich seiner Reichweite agiert? Verbindet man damit einen Entwicklungstrend in der modernen Wissenschaft, der hin zu einem Verschwinden der Grenzen zwischen den Disziplinen und dann zu einer neuen Einheit der Wissenschaften geht? Oder handelt es sich eher um einen Dachbegriff für ein Forum, welches die zukünftigen Auswirkungen von Wissenschaft und Technik erkundet?

$\mathrm{Zu}$ allen hier skizzierten Rahmungen lassen sich Debattenbeiträge finden, in denen die Autoren den Begriff CT unterschiedlich konzeptualisieren. Zum einen sind inzwischen eine ganze Reihe von Foresight-Berichten und Innovations-Reports veröffentlicht worden, die sich den technisch-ökonomischen $\mathrm{Zu}$ kunftsperspektiven von Converging Technologies oder den zentralen Bestandteilen der zugehörigen Technologiebereiche und ihren Interdependenzen widmen (z.B. EGE 2005). Ein zweiter

\section{Was sind Converging Technologies?}

Aufgrund dieser Situation hat sich bisher keine einheitliche Definition von Converging Technologies herausgebildet. In gewisser Weise folgt CT damit der Geschichte der Nanotechnologie, die ebenfalls mit derartigen definitorischen Schwierigkeiten konfrontiert war und ist. Auch bei dieser lassen sich Definitionen finden, die eher eine enge natur- und ingenieurwissenschaftliche Rahmung verfolgen, und solche, bei der Nanotechnologie eher als Synonym für jegliche moderne Wissenschaft und Technik, also als Avantgarde-Label gefasst wird.

\section{Literatur}

EGE - The European Group on Ethics in Science and New Technologies to the European Commission (eds.): Ethical aspects of ICT implants in the human body. Luxemburg 2005. the conditio humana, and search for orientation. In: Futures 39/2007, S. 380-392.

HLEG - High Level Expert Group „Foresighting the New Technology Wave“: Converging Technologies - Shaping the Future of European Societies. Brüssel 2004.

Quendt, Chr. / Fleischer, T. / Rader, M.: Converging Technologies als Gegenstand internationaler forschungspolitischer Debatten - Beobachtungen, Analysen, Probleme. In: Bora, A. / Bröchler, St. / Decker, M. (Hrsg.): Technology Assessment in der Weltgesellschaft. Berlin 2007, S. 213-220.

Roco, M.C. / Bainbridge, W.S.: Converging Technologies for Improving Human Performance, National Science Foundation. Arlington 2002.

Schmidt, J.C.: Knowledge Politics of Interdisciplinarity: Specifying the Type of Interdisciplinarity in the NSF's NBIC Scenario. In: Innovation 20, 4/2007, S. 313-328.

\section{AUTOREN + KONTAKT}

Torsten Fleischer und Michael Rader sind Mitarbeiter am Institut für Technikfolgenabschätzung und Systemanalyse (ITAS).

ITAS, Karlsruher Institut für Technologie (KIT), Campus Nord, Hermann-von-Helmholtz-Platz 1, 76344 Eggenstein-Leopoldshafen.
Grunwald, A.: Converging Technologies: Visions, increased contingencies of 
(c) 20I0 Authors; licensee IÖW and oekom verlag. This is an article distributed under the terms of the Creative Commons Attribution Non-Commercial No Derivates License (http://creativecommons.org/licenses/by-nc-nd/3.o/), which permits unrestricted use, distribution, and reproduction in any medium, provided the original work is properly cited. 